\title{
Comunidades de práctica e innovación: aprender a emprender en el área de Bibliotecología y Ciencias de la Documentación
}

\author{
Rosario Arquero Avilés * \\ Gonzalo Marco Cuenca \\ Silvia Cobo Serrano \\ L. Fernando Ramos Simón
}

Artículo recibido:

19 de agosto de 2013.

Artículo aceptado:

7 de febrero de 2014.

\section{Resumen}

El propósito del trabajo es ofrecer directrices teóricas y metodológicas para diseñar, crear y desarrollar Comunidades de Práctica (CoP) en el área de la Bibliotecología y las Ciencias de la Documentación. Las CoP presentan la posibilidad de integrar en un mismo entorno o comunidad a miembros con objetivos comunes que buscan beneficiarse de la generación de conocimientos compartidos. En este contexto de desarrollo se presenta un caso real, circunscrito en el marco de un proyecto de innovación educativa, con alumnos del Máster en Gestión de la Documentación, Bibliotecas y Archivos de la Facultad de Ciencias de la Documentación de la Universidad Complutense de Madrid (España). Dicho

Los cuatro autores pertenecen a la Universidad Complutense de Madrid. España. Contacto: carquero@pdi.ucm.es

INVESTIGACIÓN BiBLIOTECOLÓGICA, Vol. 28, Núm. 63, mayo/agosto, 2014, México, ISSN: 0187-358X. pp. 193-222 
caso ofrece una perspectiva de uso efectivo de una CoP aportando un escenario o red de intercambio de conocimiento común, que sirve de apoyo al aprendizaje educativo y cuya finalidad principal es fomentar la cultura innovadora y emprendedora entre todos sus participantes.

Palabras clave: Planificación y gestión de proyectos documentales; Comunidades de Práctica (CoP); Bibliotecas universitarias; Innovación educativa en Bibliotecología y Ciencias de la Documentación; Universidad Complutense de Madrid (España); Estudio de caso.

\section{Abstract}

Practice and innovation communities: learning to take action in the field of Library and Documentation Science

Rosario Arquero-Avilés, Gonzalo Marco-Cuenca, Silvia Cobo-Serrano and L. Fernando Ramos-Simón

This study aims to provide guidelines for designing, creating and developing a Community of Practice $(\mathrm{CoP})$ in the area Library and Documentation Science. Such a CoP offers the potential of bringing together persons with shared interests and objectives who can reap real benefits through the generation of shared knowledge. The paper presents a case study involving an educational project with master degree candidates in the Documentation, Libraries and Archives Management of the Faculty of the Documentary Science of the Universidad Complutense de Madrid (Spain). This case provides a view of the effective use of CoP, which constitutes a scenario or network for exchange of shared knowledge, while underpinning educational learning and promoting the culture of innovation and enterprise among the members of the community.

Keywords: Planning and Management of Documentation Projects; Communities of Practice; University Libraries; Educational Innovation in Library and Documentation Sciences. 


\section{INTRODUCCIÓN}

T as Comunidades de Práctica (CoP) se han considerado, en los últimos Laños, como una teoría potencial para la creación de conocimiento desde una perspectiva colaborativa (Roberts, 2006; Pan \& Leidner, 2003). Las CoP muestran como objetivos básicos la colaboración en grupo y la generación de conocimiento compartido. Como grupo social, sus miembros se unen por medio de un compromiso mutuo de colaboración y se focalizan en una materia, actividad o interés determinado para intercambiar conocimiento y aprender de una forma práctica. En tal situación, se percibe que los principales activos de la red son el conocimiento y las buenas prácticas y ambos se pueden adquirir a partir del intercambio de experiencias, recursos e información entre sus miembros.

En esta línea, la Facultad de Ciencias de la Documentación de la Universidad Complutense de Madrid ha desarrollado un proyecto denominado CoP-Innova, dentro de los principios del Espacio Europeo de Educación Superior (EEES) que, como compromiso político europeo, fue adoptado formalmente en la denominada Declaración de Bolonia (European Ministers of Education, 1999). La implantación del EEES ha supuesto un cambio estructural que incide en un modelo de enseñanza centrado en el proceso de aprendizaje de los estudiantes, claramente dirigido a la adquisición y desarrollo de competencias, así como a los procedimientos para su evaluación. Para responder al reto del EEES, la Universidad Complutense de Madrid ha impulsado la iniciativa "Convocatoria de Proyectos de Innovación y Mejora de la Calidad Docente", cuya finalidad fundamental es fomentar la innovación educativa con objeto de lograr una docencia universitaria de calidad sobre el eje fundamental del EEES, según el cual el proceso de aprendizaje del estudiante debe enriquecerse con la innovación tanto de clases teóricas como de prácticas.

La obtención de un proyecto en el marco de dicha convocatoria supone una apuesta de la Universidad Complutense por la institucionalización de buenas prácticas docentes en el proceso de enseñanza-aprendizaje; en ella se valoran aspectos como los siguientes:

- La originalidad de la propuesta.

- El interés del proyecto para la mejora del proceso de docencia-aprendizaje.

- La contribución del proyecto al establecimiento y difusión de buenas prácticas docentes.

- La experiencia y formación del grupo de trabajo en materia de innovación educativa y de desarrollo de los recursos de las Tecnologías de la Información y de la Comunicación en la enseñanza. 
- El progreso y sostenibilidad de la implantación de los proyectos.

- La amplitud del ámbito de aplicación del proyecto, en particular el número de estudiantes reales que pueden beneficiarse del proyecto, la aplicabilidad de los resultados a otras asignaturas o titulaciones y el grado de interdisciplinariedad.

- La adecuación de los recursos solicitados en relación con el plan de trabajo, la metodología y los objetivos.

- La consistencia del proyecto, en particular la adecuación de los objetivos con la metodología propuesta y el cronograma fijado.

El proyecto de innovación, cuya metodología y resultados presentamos en este trabajo, se circunscribe en dicha iniciativa y se ha basado en la conformación de una comunidad de práctica denominada CoP-Innova, que implica un doble sentido: por un lado, nos encontramos con la comunidad de práctica como estructura que facilita el compartir conocimiento y hacerlo circular (Vásquez, 2011) y, por otro, aparece la innovación como un elemento que implica la generación de una cadena de valor que se manifiesta en tres fases: la generación de la idea, su concreción y su difusión (Hansen \& Birkinshaw, 2007). Las CoP son consideradas además como un enclave para el aprendizaje activo y como incubadoras de la innovación (Holly, 2004). No obstante, para que las ideas puedan ser llevadas a buen puerto en forma de productos, servicios u otro elemento de valor han de ser formalizadas y, por ello, CoP-Innova toma como base los principios y fundamentos de la gestión de proyectos con la intención de promover propuestas, en forma de proyectos y adaptadas a un escenario piloto, cuya viabilidad pueda determinar su aplicabilidad en circunstancias reales.

\section{El contexto teórico de CoP-Innova: la gestión de proyectos innovadores en unidades de información y documentación}

La asignatura Gestión de Proyectos en Unidades Documentales, de carácter obligatorio dentro del Máster en Gestión de la Documentación, Bibliotecas y Archivos de la Facultad de Ciencias de la Documentación de la Universidad Complutense de Madrid y en la que se ha desarrollado la experiencia del proyecto CoP-Innova, se ha focalizado en el estudio de la aplicación de técnicas de gestión de proyectos por parte del profesional de la información y documentación, relacionada con la planificación de este tipo de unidades.

En este sentido, la gestión de proyectos se sitúa en el dominio del management de unidades de información y documentación y, en concreto, en el ámbito de su planificación. Tradicionalmente han sido numerosos los autores 
que han descrito la planificación como una función del management de las organizaciones en general (Drucker, 2002; Bueno Campos, 1996) y del management de las unidades de información y documentación en particular (Koontz \& O’Donnell, 1972; McClure \& Samuels, 1982; Lynch, 1985; Bryson, 1997 y 1999; Koontz y Weihrich, 1994; Prytherch, 1994; Evans \& Layze11, 2003; Stueart \& Moran, 2002; Ramos Simón, 1995 y 2003; Pacios Lozano, 1997, entre otros).

Este vínculo entre la gestión de proyectos y la planificación en el ámbito de conocimiento del management explica que la formulación de proyectos en unidades de información y documentación se circunscriba plenamente en su planificación operativa. En este sentido, se parte de considerarla como aquella planificación directamente relacionada con la estratégica, subordinada a la misma y situada jerárquicamente en un nivel inferior. Esto significa que la planificación estratégica o a largo plazo establece las grandes líneas de acción que una unidad de información y documentación deberá seguir para caminar hacia un futuro previsible, mientras que la planificación operativa deberá traducir las directrices generales de los planes estratégicos a un nivel más detallado e inmediato con la finalidad principal de obtener resultados (Arquero \& García-Ochoa, 2005: 34).

Una vez identificado el ámbito de conocimiento marco, hemos de destacar la existencia de una bibliografía de referencia seleccionada sobre la materia que conforma la base científica y conceptual de nuestra revisión y análisis en lo que incumbe a la aplicación de principios y metodología propios de la gestión de proyectos.

En esta línea, podemos citar obras de carácter monográfico desde la década de 1990 que han situado la gestión de proyectos como parte fundamental e imprescindible dentro del área de Bibliotecología y Ciencias de la Documentación. Se pueden conocer las características principales de dichas publicaciones mediante un breve y conciso recorrido evolutivo de las mismas a partir del establecimiento de dos grandes periodos cronológicos: de 1990 al año 2000 y de 2001 hasta la actualidad.

En el primer periodo es preciso destacar los trabajos especializados en dicha temática de MacLachlan (1996) y Black (1996). En conjunto, sus publicaciones detallan, por un lado, las diferentes etapas o fases del ciclo de vida de los proyectos y, por otro, explican la necesidad de realizar estimaciones de tiempo, presupuesto, riesgos y recursos humanos.

Del segundo periodo cronológico hemos de subrayar varios trabajos. Citamos en primer lugar la aportación de Allan (2004), que proporciona una guía de recursos para la gestión de proyectos en todos los tipos de servicios de información mediante métodos de probada eficacia. Los estudios de Carpenter 
(2008 y 2011) exponen las fases y actividades pertinentes para la gestión de un proyecto y abordan temas tales como la formulación de objetivos del proyecto, su alcance, la repercusión que tiene la cooperación de los recursos humanos de la unidad de información en la gestión de un proyecto así como los temas y programas de financiación. Cabe mencionar también, por su repercusión innovadora, el trabajo de Fagan (2009) donde se aborda la gestión de proyectos web para bibliotecas académicas y se realiza un repaso a la importancia y función del gestor del proyecto, la definición del proyecto, el equipo y diversos aspectos relacionados con la comunicación interna y externa.

Al contenido y enfoque de las publicaciones monográficas a las que nos hemos referido hemos de añadir la revisión de una serie de publicaciones adicionales que presentan el enfoque de aplicación que entronca con la propia orientación de revisión bibliográfica de este epígrafe y de aplicación práctica del proyecto CoP-Innova. Tales publicaciones recogen experiencias prácticas de aplicación de gestión de proyectos en unidades de información y documentación y las podemos sistematizar en los bloques o categorías temáticas siguientes: digitalización (DeRidder, 2007; Fenton, 2007; Henshaw, 2011; Holley, 2004; Hull \& Dreher, 2001; Levi, 2010; Londhe, Desale \& Patil, 2011; Middleton, 1999; Müller \& Maurer, 2011; Saikia \& Kalita, 2011; Sakaguchi, Shimada \& Wasserstrom, 2010), fomento de la lectura (Roselló, 2006; Train \& Elkin, 2001), formación de usuarios (Betty, 2008; Murphy, 2008), herramientas de gestión de contenidos (Hayman, 1997; Koontz et al., 2004; Medawar, 2007; Sharpe \& Vacek, 2010), operaciones técnicas (Anderson, 1998; Martey, 2002), recursos humanos (Anzalone, 2000), repositorios institucionales y otros recursos digitales (Barwick, 2007; Chellapandi, Han \& Boon, 2010; Greene, 2010; Piorun \& Palmer, 2008), señalización bilingüe de bibliotecas (Evans, 2011), servicios virtuales de referencia (Burich et al., 2006; Clements, 2009; Duncan \& Gerrard, 2011) y sistemas integrados de gestión bibliotecaria (Adeyoyin \& Akinyosoye, 2004; Darko-Ampem, 2006; Evans \& Thomas, 2007; Lewis, 1995; Otunla \& Akanmu-Adeyemo, 2010).

En conjunto, de la revisión de las publicaciones especializadas en la materia podemos derivar una serie de rasgos que constituirían el núcleo de elementos básicos en los que se sustenta la gestión de proyectos y su aplicación en CoP-Innova:

- Comprende el desarrollo de etapas sucesivas e identificables (planificación, diseño, ejecución, control o seguimiento y evaluación) (Webb, 2001; Winston \& Hoffman, 2005).

- Los proyectos se basan en objetivos concretos (Rosacker, 2010) y, junto con las fases, requieren de la aplicación de ciertas herramientas, procedimientos y conocimientos específicos (Moore, 1998). 
- El aspecto temporal y cualitativo así como los recursos económicos son fundamentales y deben estar presentes en la gestión de un proyecto. Así lo manifiesta Pat Wagner: "la gestión de proyectos consiste en finalizar el trabajo a tiempo, por debajo del presupuesto y con la calidad prevista" (2006: 24). Otro autor que constata este planteamiento es Roger Atkinson, quien alude en su obra a la norma británica Gestión de proyectos BS60794 para señalar como elementos esenciales en la gestión de un proyecto "la motivación para lograr los objetivos del proyecto a tiempo y los costes, calidad y el rendimiento específicos" (1999:338).

- El éxito de un proyecto bien gestionado desde un punto de vista técnico motiva a los profesionales de las unidades de información y documentación para realizar otras actividades de mayor complejidad y, además, despierta su interés por el trabajo en grupo y por la cultura del trabajo por proyectos dentro de las organizaciones (Winston \& Hoffman, 2005).

En síntesis, se puede establecer que la gestión de proyectos comprende la aplicación de un conjunto de técnicas, herramientas, conocimientos y habilidades para el cumplimiento de las actividades programadas, con la finalidad de alcanzar los objetivos de un proyecto específico en un tiempo limitado. En este sentido, un proyecto se concibe como un proceso con un principio y un final definidos, que conduce a la generación de un producto, servicio, actividad o resultado concreto y que, además, implica planificar la formulación, el desarrollo y la conclusión del mismo.

En línea con dichas consideraciones se presenta un modelo de proyecto-tipo en unidades de información y documentación en el que se ha sustentado la aplicación de la base teórica y metodológica en gestión de proyectos de CoP-Innova y que integra los siguientes elementos y principios:

- El objeto de un proyecto es obtener un resultado único (en la unidad de información y documentación este resultado se entiende como un producto, servicio y/o actividad documental).

- Dicho producto, servicio y/o actividad es generado y proyectado para un destinatario (es muy común que los destinatarios sean los propios usuarios de la unidad de información y documentación o los recursos humanos que trabajan en la misma).

- La obtención del resultado implica la asignación de recursos humanos, económicos, materiales y técnicos; además, su desarrollo se tiene que ejecutar en un tiempo limitado (asociado a un cronograma y a unos plazos). 
- Estos productos, servicios y actividades documentales han de ser planificados, ejecutados y evaluados; es decir, se pueden gestionar aplicando las fases del ciclo de vida de un proyecto: inicio, planificación, ejecución y control, y cierre.

\section{El contexto institucional y estratégico de referencia}

La Universidad Complutense es una institución cuyos orígenes se sitúan más de cinco siglos atrás. Fue fundada mediante Bula Pontificia concedida por el Papa Alejandro VI en 1499 (Universidad Complutense de Madrid, 2002). Se trata de una de las universidades europeas y españolas con más tradición e historia. Como dato significativo, téngase en cuenta que en el curso 15091510 ya funcionaban cinco Facultades: Artes y Filosofía, Teología, Derecho Canónico, Letras y Medicina.

En la actualidad se estructura en dos campus principales: el campus de Moncloa y el campus de Somosaguas. Cuenta con más de 80000 alumnos matriculados, más de 6000 profesores y oferta 274 titulaciones oficiales diferentes (estudios de grado, posgrado y doctorado) y 187 titulaciones propias (estudios de Magíster, Especialista y Experto) (Universidad Complutense de Madrid, 2013).

En dicho contexto institucional global, la Biblioteca de la Universidad Complutense (BUC) se perfila como una unidad funcional al servicio de la docencia y la investigación. La BUC es considerada la primera biblioteca universitaria de España y la segunda biblioteca de dicho país (con más de 2700 000 volúmenes), después de la Biblioteca Nacional española.

La estructura de la Biblioteca de la Universidad Complutense es la siguiente (Biblioteca de la Universidad Complutense de Madrid, 2013):

- Dirección de la BUC.

- Servicios Centrales. Ejercen la responsabilidad de la unificación de los criterios, la coordinación y normalización de los procesos y servicios de las bibliotecas de los distintos centros de la Universidad Complutense (Facultades, Escuelas e Institutos de Investigación).

- Biblioteca Histórica "Marqués de Valdecilla". Encargada de la gestión, mantenimiento, conservación y difusión del patrimonio histórico bibliográfico de la Universidad Complutense.

- Bibliotecas de Centros. Tienen la responsabilidad del impacto de los servicios bibliotecarios sobre la comunidad universitaria. Se trata de un total de 34 bibliotecas interconectadas a través de la red de datos de la Universidad Complutense de Madrid. Desde cualquiera de ellas 
se puede acceder al catálogo general y utilizar indistintamente sus servicios.

En el marco de dicha estructura, la Biblioteca de la Facultad de Ciencias Económicas y Empresariales es una biblioteca de centro, pero al mismo tiempo cumple la función de Biblioteca Coordinadora del Campus de Somosaguas. La doble condición de dicha biblioteca aportó unos requisitos relevantes y significativos para la elección del escenario de pruebas y pilotaje llevado a cabo a partir de la experiencia de CoP-Innova, y a cuya creación, estructura y características nos referimos en el apartado siguiente de nuestro artículo. Esta biblioteca, seleccionada como biblioteca piloto, ha proporcionado la base del entorno de aplicación práctica y profesional de CoP-Innova, teniendo siempre presente la vinculación establecida en el epígrafe anterior entre la gestión de proyectos y la planificación en el ámbito de conocimiento del management; asimismo, nos hemos centrado en su relación con las seis líneas estratégicas del Plan Estratégico 2010-2013 (Biblioteca de la Universidad Complutense de Madrid, 2010): Personas, Organización y Calidad, Financiación e Infraestructuras, Servicios, Biblioteca y Sociedad y, por último, Colecciones. Queda excluida la séptima línea estratégica denominada Patrimonio Bibliográfico, reservada a temas de conservación y difusión de las colecciones bibliográficas antiguas. El criterio de exclusión de dicha línea se debe a que todo el fondo anterior al siglo XIX de la Biblioteca de la Facultad de Ciencias Económicas y Empresariales está gestionado por la Biblioteca Histórica "Marqués de Valdecilla", que como se ha señalado está especializada en fondo antiguo.

\section{Material y métodos. Creación, ESTRUCTURA Y CARACTERÍSTICAS DE LA COP}

En lo que se refiere a las directrices y elementos para la conformación y desarrollo de CoP-Innova como comunidad de práctica, se ha partido del concepto acuñado por Wenger \& Lave (1991) según el cual el aprendizaje implica la participación en una comunidad y la adquisición de conocimiento se considera un proceso de carácter colectivo. Los autores añaden que las comunidades de práctica son grupos de personas que comparten una preocupación o pasión por algo que realizan y que, en la medida en que interactúan entre sí de forma regular, aprenden como mejorar esta actividad, para lo cual requieren centrarse en un tema de interés, un foco o una tarea específica a realizar.

En este sentido, la conformación de CoP-Innova se ha sustentado en las tres premisas o dimensiones fijadas por Wenger E. (1998) (Figura 1): 
- Mutual Engagement (compromiso mutuo). Todos los integrantes de la CoP comparten su propio conocimiento y reciben el de los otros (es decir, todos tienen algo que enseñar y todos tienen algo que aprender).

- Joint Enterprise (iniciativa conjunta). La CoP debe contar con unos objetivos comunes, con independencia de que los miembros tengan intereses y necesidades distintos.

- Shared Repertoire (repertorio compartido). Se refiere al conjunto de recursos comunes de la CoP (terminología, procedimientos, herramientas, maneras de hacer o conceptos que la CoP produce o adopta en el curso de su existencia y que pasan a formar parte de su práctica).

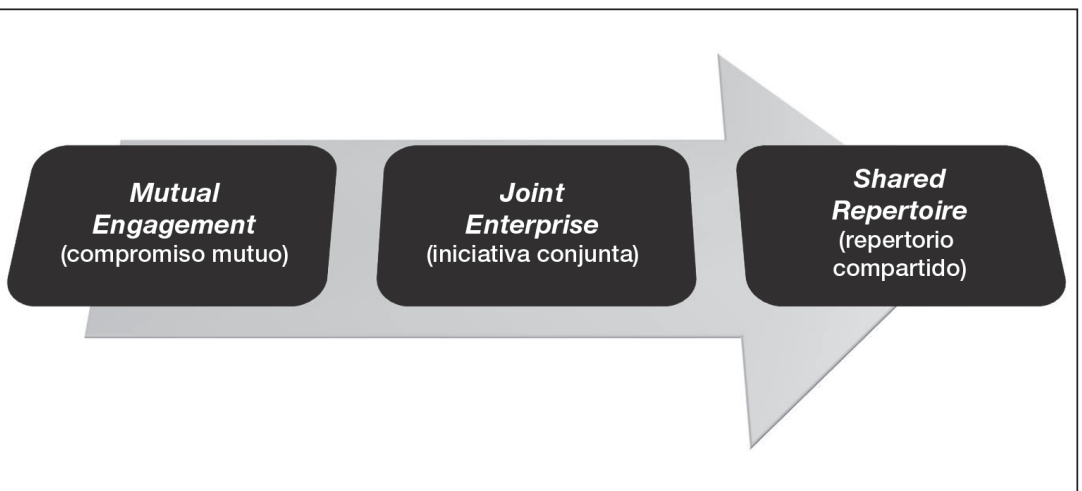

Figura 1. Dimensiones fijadas por Etienne Wenger para una CoP.

Este proyecto ha considerado la constitución de la CoP como una estrategia de colaboración en la que se combinan las perspectivas del trabajo, el aprendizaje y la innovación entre los miembros integrantes, que proceden de diferentes sectores de la Universidad (Gongla \& Rizzuto, 2001; Palincsar \& Herrenkohl, 2002). Continuando en esta misma dirección, la CoP se concibe (como sugieren Lesser \& Starck, 2001) como un medio para contrarrestar el efecto que tienen en las organizaciones los diferentes niveles jerárquicos y para obtener importantes beneficios para nuestra organización (en concreto para la planificación y la gestión del cambio de sus bibliotecas sobre la base de la experiencia piloto desarrollada). En esta línea, la CoP en la que se ha sustentado el desarrollo de nuestro proyecto de innovación ha servido para generar ideas de nuevos productos y servicios (Lesser \& Everest, 2001), concebidos como proyectos en su propuesta de formulación para un contexto real.

La estructura de CoP-Innova (Figura 2) se basa en la integración de tres elementos clave: la aportación de conocimientos técnicos y metodológicos sobre la gestión de proyectos en unidades de información y documentación, 
un contexto real de aplicación de la experiencia colaborativa y la contribución de ideas transformadas en proyectos de innovación. La combinación de dichos elementos se ha materializado en una experiencia real de integración de los actores que han conformado la CoP: profesores, investigadores, bibliotecarios profesionales y alumnos, como integrantes y beneficiarios directos de la misma (Figura 3).

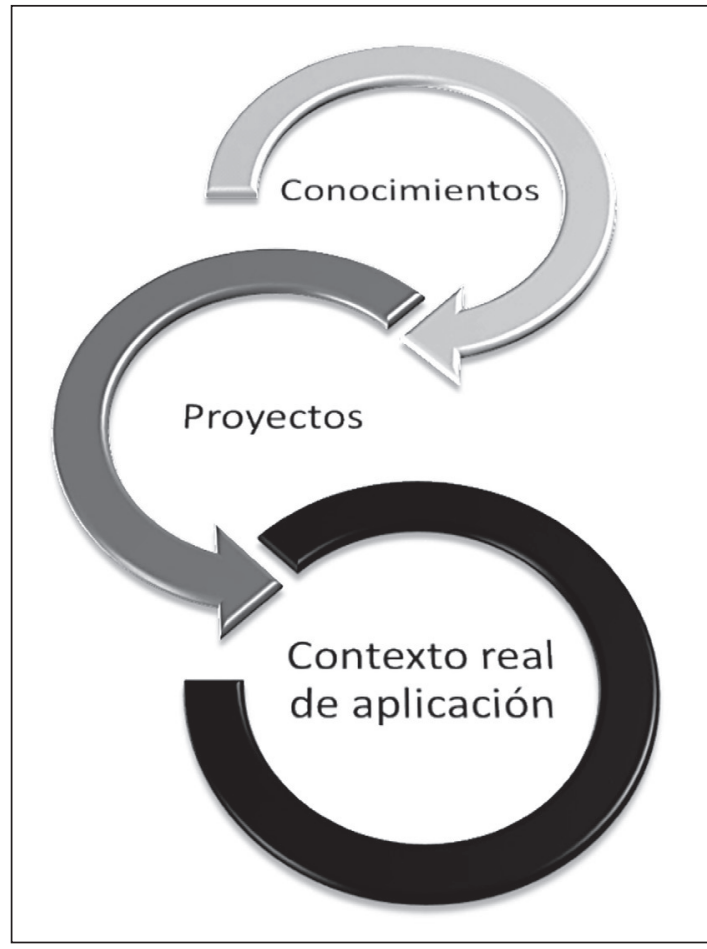

Figura 2. Elementos clave de la estructura de CoP-Innova.

El sector de los profesores e investigadores se integra por:

- Un responsable del proyecto CoP-Innova que ha ejercido de gestor de la CoP (CoP-manager). La existencia de un CoP manager responde a la función de facilitar y hacer posible que el resto de miembros de la CoP lleguen a acuerdos sobre los problemas o cuestiones relevantes (Gongla \& Rizzuto, 2001). El CoP-manager ha de fomentar además la interacción facilitando la correcta interpretación de las diferentes contribuciones del resto de miembros de la comunidad (Handley, Sturdy, Fincham \& Clark, 2006). El CoP-manager también ejerce la labor de 
supervisión y coordinación en el desarrollo de las actividades y tareas desempeñadas por los participantes (Brown \& Duguid, 1991) y, en última instancia, es responsable final de los resultados derivados del funcionamiento de la $\mathrm{CoP}$ (incluida la elaboración y entrega de la memoria final). En nuestro proyecto este rol ha sido desempeñado por la profesora titular de la asignatura en la que se ha desarrollado el proyecto de innovación.

- Un equipo de proyecto (Project Team) del sector de profesores e investigadores. Este equipo ha ejercido el rol de apoyo y/o asesoramiento al CoP-manager en la preparación de las directrices técnicas y metodológicas de trabajo, al igual que en la gestión y seguimiento de la propia $\mathrm{CoP}$ y del proyecto en su conjunto. Se trataría de consultores del ámbito académico e investigador que han reportado a la CoP-manager como apoyo para la consecución de los objetivos establecidos. El equipo de proyecto de este sector fue constituido por los siguientes miembros: un profesional asociado, consultor externo y experto en proyectos; un investigador y un profesor titular experto en Economía de la Información.

El rol de aportación del contexto real de aplicación de las propuestas de proyectos en el ámbito profesional ha correspondido al sector de profesionales bibliotecarios del equipo de proyecto, liderado por la Directora y la Subdirectora de la Biblioteca de la Facultad de Ciencias Económicas y Empresariales.

El sector de alumnos de CoP-Innova se conformó por los alumnos de posgrado matriculados en el curso académico, tanto en turno matutino como vespertino, con un total de 47 alumnos participantes distribuidos en 17 equipos de trabajo.

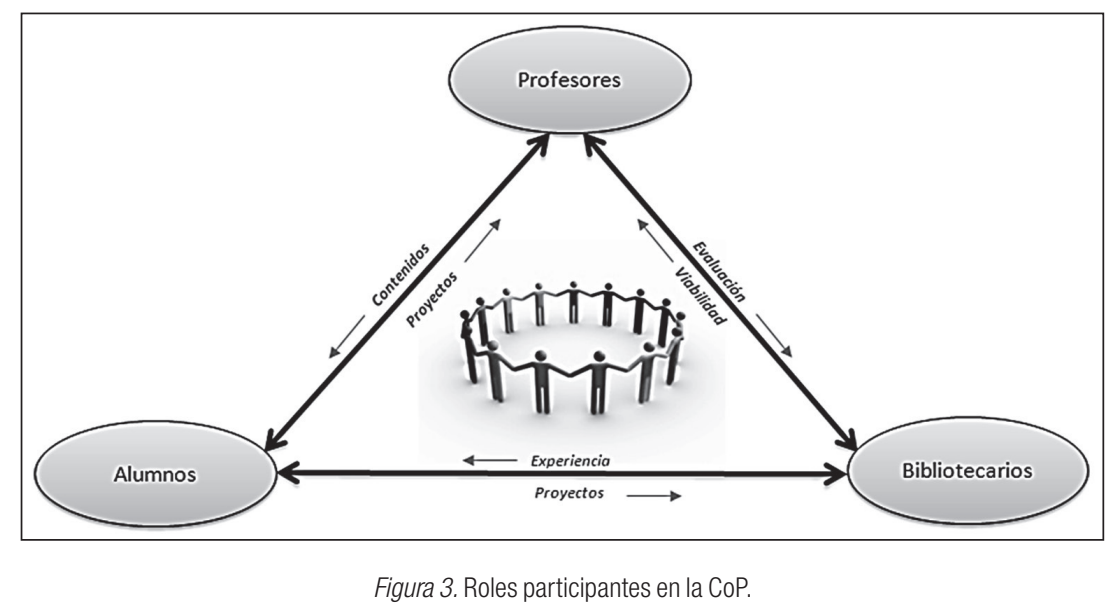


CoP-Innova también ha contado con una serie de herramientas de apoyo a la comunicación, participación social y trabajo colaborativo, como un campus virtual basado en la aplicación web Moodle, que cuenta con los módulos de administración, tareas, consulta, foro, recursos, sindicación RSS y Atom, correo electrónico e incluso un módulo wiki, y un grupo virtual privado en la red Facebook. Destaca también que por medio del campus virtual se han podido obtener estadísticas de participación de todos los miembros, lo que proporciona información al CoP-manager para la gestión de la comunidad, generación de alertas, fidelización de miembros, reactivación de actividades, etc. Como herramientas exclusivas de gestión de proyectos se ha utilizado principalmente MS Project 2010.

\section{Funcionamiento de la CoP}

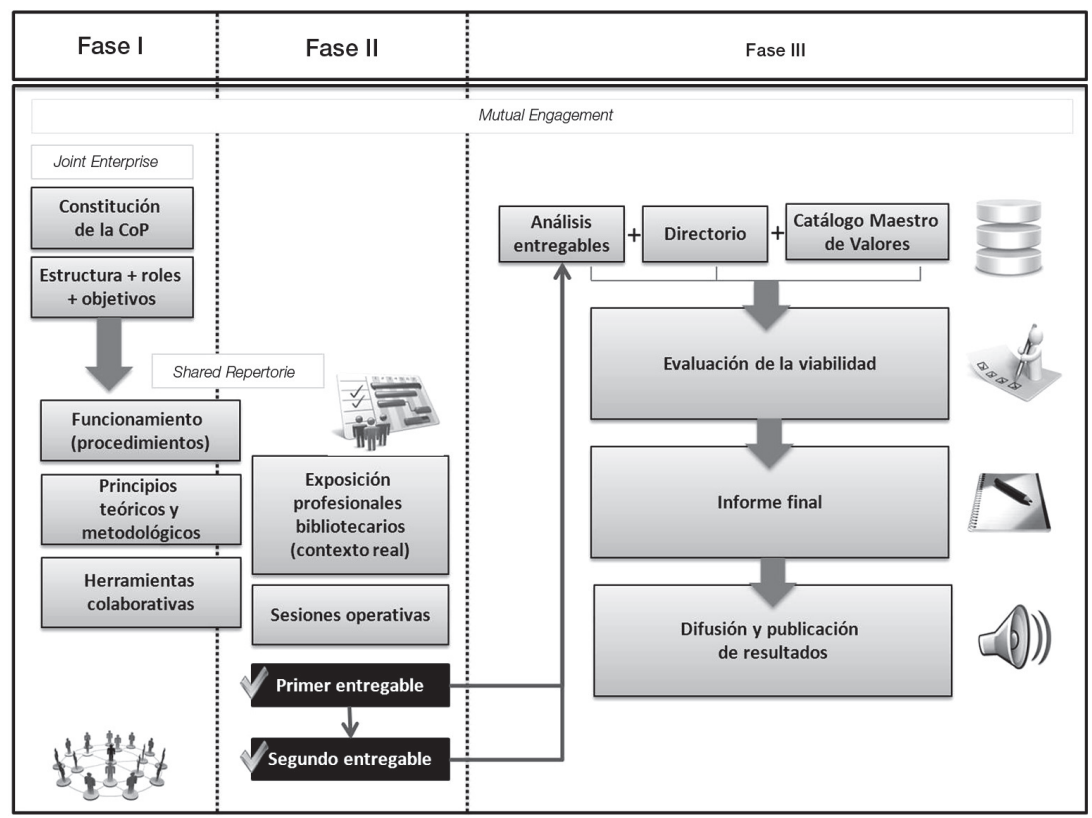

Figura 4. Modelo de funcionamiento de CoP-Innova.

El funcionamiento de la CoP se basa en el modelo descrito en la Figura 4 y en las fases y metodología de trabajo siguientes:

Primera fase. Se comparten las bases del Mutual Engagement (compromiso mutuo) y se acomete el proceso de formación de CoP-Innova, la definición de su estructura, la identificación de roles de sus integrantes y el establecimiento de sus objetivos (Joint Enterprise-iniciativa conjunta). Se establecieron a continuación 
las bases del Shared Repertoire (repertorio compartido) de la CoP por parte del equipo de proyecto del sector de profesores e investigadores, basado en:

- La fijación de principios de funcionamiento de la CoP.

- La exposición de principios teóricos y metodológicos para la planificación y gestión de proyectos en unidades de información y documentación, sobre la base de los objetivos globales de la asignatura en la que se ha desarrollado el proyecto CoP-Innova:

- Conocimiento de los principios teóricos y metodológicos para la planificación y gestión de proyectos en unidades de información y documentación.

- Comprensión y aplicación de la metodología base en la que se sustenta la planificación y la gestión de proyectos en unidades de información y documentación.

- Búsqueda de la interacción entre dicha metodología y los procesos documentales concebidos como proyectos y análisis de su implementación en bibliotecas universitarias.

- Presentación de herramientas y espacio colaborativo.

La metodología utilizada en esta fase se basó en exponer a los alumnos los contenidos derivados del análisis y sistematización de las aportaciones analizadas en el apartado referente al contexto teórico de CoP-Innova, en la adaptación de dicha base conceptual a las directrices propuestas en la asignatura y en su aplicación específica el contexto real de la biblioteca piloto de CoP-Innova.

Segunda fase. Se completaron las bases del Shared Repertoire (repertorio compartido) con las aportaciones del equipo de proyecto del sector de profesionales bibliotecarios que en síntesis se basaron en la exposición de un conjunto de buenas prácticas: competencias y funciones, compromisos de calidad, procedimientos, servicios y productos que se realizan en la biblioteca piloto y la identificación de las líneas estratégicas del Plan Estratégico 2010-2013 de la Biblioteca de la Universidad Complutense de Madrid.

En esta misma fase se ponen en práctica estrategias para facilitar la colaboración e interacción entre los diferentes integrantes de la CoP (sesiones de lluvia de ideas, discusiones grupales, actividades en línea, foros, presentaciones y exposiciones de casos y resultados previos, entre otros) que buscan canalizar la presentación inicial de propuestas de proyectos innovadores por parte de los diferentes equipos de trabajo. Dichas propuestas iniciales se materializaron en un primer entregable que debía presentar cada equipo y que respondía al esquema siguiente: 
a) Prospección. Síntesis del funcionamiento y características de la unidad de información para la que se propone el proyecto en el marco de su plan estratégico y búsqueda y revisión de experiencias externas similares al proyecto que se propone.

b) Análisis $\mathrm{SWOT}^{1}$ de la propuesta de proyecto.

c) Elección de la línea o líneas estratégicas en la que se incluye la propuesta.

d) Formulación de la finalidad global del proyecto, necesidades a las que responde y principales beneficios derivados.

e) Identificación de los stakeholders del proyecto.

Por último, se lleva a cabo la formulación de propuestas finales de acuerdo con los elementos constitutivos del repertorio compartido y la materialización final de dichas propuestas por parte de los diferentes equipos en un segundo entregable que respondió al esquema que mostramos en la Figura 5. Tercera fase. Consiste en el análisis de documentos (específicamente de los informes de los proyectos propuestos) y la creación un directorio final de los mismos. ${ }^{2}$ Se definió también un Catálogo Maestro de Valores (CMV), que es la herramienta de cálculo que facilita datos estadísticos con el objetivo de servir de complemento adicional a la evaluación cualitativa realizada por los expertos; fue diseñada con el programa ms Excel 2010 y ha permitido el análisis en conjunto de los diferentes entregables del proyecto tomando como base la asignación de valores para cada variable de un entregable. Esencialmente, se trata de un instrumento básico de evaluación cuantitativa que permite realizar lecturas, agregadas o específicas, a partir de los datos generados. Los valores permiten agrupar y clasificar criterios comunes, aportando un conjunto mínimo de datos normalizados que facilita la explotación de tablas y gráficos dinámicos. Cada valor se corresponde con un descriptor, identificado por un código unívoco, que aporta el significado aceptado para cada concepto. Algunos valores incluyen a su vez otros valores de nivel jerárquico inferior, lo que ha permitido el análisis de los datos a varios niveles de detalle. Para los datos relacionados con costes (presupuesto) y tiempo se ha usado una escala de Likert basada en tres criterios: alto, medio y bajo.

Durante esta etapa se evaluaron y discutieron las propuestas finales de proyectos con vistas a determinar su viabilidad para una posible implementación real, con especial atención a aquellos que pudieran ser reutilizables y exportables a otras bibliotecas de la Universidad Complutense y a otras

1 También conocido como análisis DAFO o FODA (Fortalezas, Oportunidades, Debilidades y Amenazas).

2 Se puede consultar un extracto de dicho directorio en el Apéndice. 
bibliotecas universitarias. En último lugar, se procedió a la redacción del informe final del proyecto de innovación así como a la difusión y comunicación de sus resultados.

\begin{tabular}{|c|c|c|c|}
\hline \multicolumn{4}{|c|}{ 1. Objetivos del proyecto } \\
\hline \multicolumn{4}{|c|}{ Alcance del proyecto y EDT (Estructura de Desglose del trabajo) } \\
\hline \multicolumn{2}{|c|}{ 2.1. Fases del proyecto } & \multicolumn{2}{|c|}{ 2.2. Relación de tareas y de hitos } \\
\hline \multicolumn{4}{|c|}{ Tiempo estimado } \\
\hline \multicolumn{2}{|c|}{$\begin{array}{l}\text { 3.1. Duración media estimada de la totalidad } \\
\text { del proyecto }\end{array}$} & \multicolumn{2}{|c|}{$\begin{array}{l}\text { 3.2 Estimación de la duración de cada una de las } \\
\text { tareas y generación del cronograma del proyecto }\end{array}$} \\
\hline \multicolumn{4}{|c|}{ Equipo del proyecto } \\
\hline \multicolumn{2}{|c|}{$\begin{array}{l}\text { 4.1. Propuesta razonada de funciones, } \\
\text { responsabilidad y roles }\end{array}$} & \multicolumn{2}{|c|}{ 4.2. Organigrama del proyecto } \\
\hline \multicolumn{4}{|c|}{ Recursos y costes estimados } \\
\hline 5.1. Personal & 5.2. Equipamiento & $\begin{array}{l}\text { 5.3. Material fungible y } \\
\text { otros gastos }\end{array}$ & $\begin{array}{l}\text { 5.4. Coste total } \\
\text { estimado }\end{array}$ \\
\hline \multicolumn{4}{|c|}{ Riesgos } \\
\hline \multicolumn{4}{|c|}{$\begin{array}{l}\text { Difusión del proyecto: audiencia, actividades, } \\
\text { canales y medios de comunicación }\end{array}$} \\
\hline \multicolumn{4}{|c|}{ Material complementario } \\
\hline \multicolumn{2}{|c|}{ 8.1. Diagrama de Gantt } & \multicolumn{2}{|c|}{ 8.2. Diagrama de red } \\
\hline
\end{tabular}

Figura 5. Elementos en un modelo de formulación de proyectos en unidades documentales (segundo entregable). 


\section{Resultados de CoP-INNova}

\section{Una comunidad de práctica viva}

Podríamos considerar que uno de los principales logros o resultados de la experiencia puesta en marcha es la vitalidad y dinamismo que la herramienta ha tenido entre sus integrantes. Las diferentes herramientas de difusión y comunicación han sido utilizadas por todos sus miembros, quienes han participado al menos una vez en alguno de los debates originados o en alguna de las líneas de discusión activas. En cuanto a esta misma participación, cabe destacar que el liderazgo del CoP-manager ha sido imprescindible para impulsar debates y precisar conceptos para que puedan ser trasladados a la operativa. Los profesionales también han jugado un papel determinante con aclaraciones sobre el escenario piloto y sus características, las funciones de sus integrantes y los productos y servicios.

El Campus Virtual y el correo electrónico del propio campus constituyeron las herramientas más usadas, pero las sesiones o foros presenciales han sido las más valoradas por los grupos de trabajo. Estos foros consistían esencialmente en la presentación de casos prácticos con intervenciones a modo de debate.

En cuanto a datos cuantitativos de actividad, la media de accesos diarios al Campus Virtual ha sido de 1.3 por participante, aproximadamente 69 accesos diarios. En datos cuantitativos globales, teniendo en cuenta la duración cuatrimestral de la asignatura, podríamos hablar de más de 5500 accesos y de una media aproximada de 104 por participante.

Los temas más tratados o más seguidos, presencialmente y en línea, han sido aquellos relacionados con las líneas estratégicas, la formulación del alcance de los proyectos y la estimación de recursos y costes.

\section{Proyectos relacionados con las líneas del plan estratégico de la Biblioteca de la Universidad Complutense}

En el apartado "El contexto institucional y estratégico de referencia" se estableció la biblioteca de la Facultad de Ciencias Económicas y Empresariales como base de presentación de propuestas de proyectos en relación con las seis líneas estratégicas del Plan Estratégico 2010-2013 (Biblioteca de la Universidad Complutense de Madrid, 2010) que afectan a la biblioteca piloto de CoP-Innova: Personas, Organización y Calidad, Financiación e Infraestructuras, Servicios, Biblioteca y Sociedad, y Colecciones.

En relación con dicho contexto y de acuerdo con el Gráfico 1, se puede manifestar que la mayoría de los proyectos presentados se relacionan 
principalmente con las líneas estratégicas Personas y Servicios, ambas representadas con un $28 \%$. Esto significa que los alumnos ven necesaria la formulación y posterior gestión de proyectos centrados en la política de personal de la biblioteca o en la formación, promoción y condiciones laborales de la plantilla de la unidad de información y documentación (es el caso de algunos proyectos planteados: teletrabajo, evaluación de puestos de trabajo, rotación de la plantilla o conciliación de la vida laboral y familiar). Además, la identificación de proyectos sobre la accesibilidad de las personas con minusvalía auditiva a los diferentes servicios y recursos de la biblioteca, la realización de estudios de usuarios a extranjeros o la creación de un repositorio institucional son acciones que reflejan la importancia de una mejora continua en los servicios bibliotecarios de las universidades.

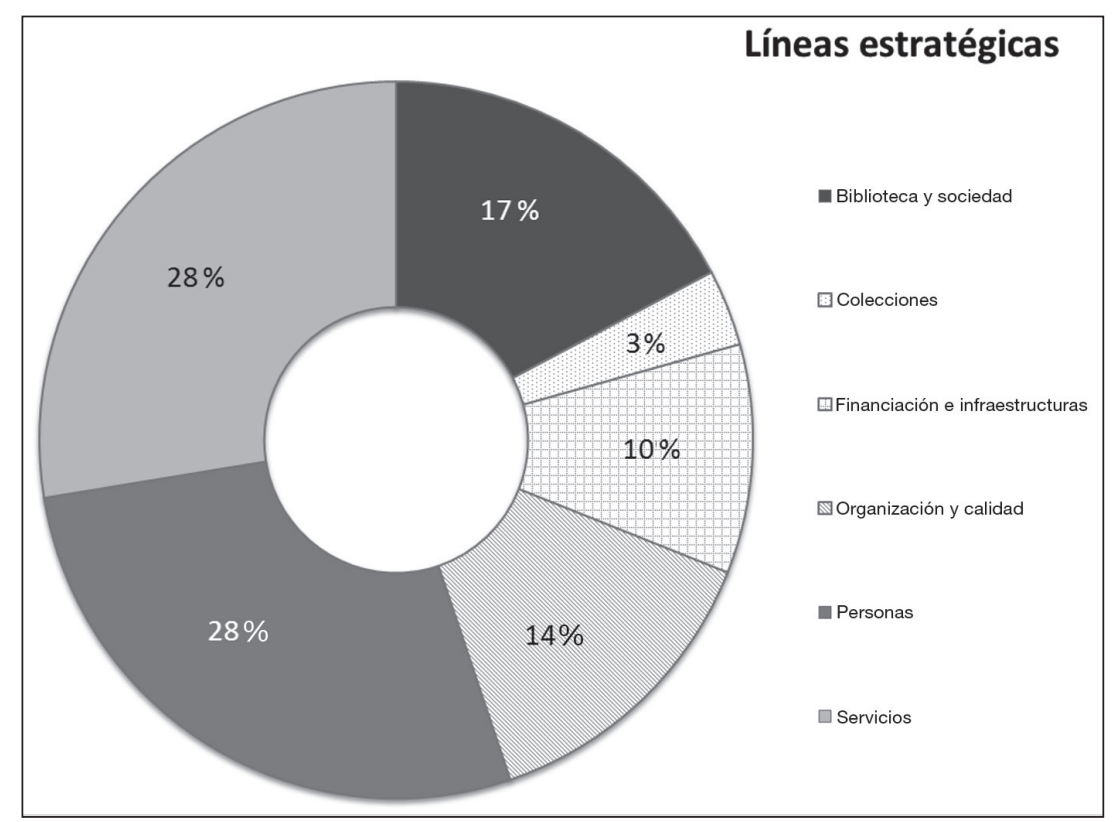

Gráfico 1. Identificación de las líneas estratégicas del Plan Estratégico 2010-2013 de la Biblioteca de la Universidad Complutense de Madrid en los proyectos propuestos por los alumnos.

En un segundo nivel, son destacables los proyectos que aluden a la línea estratégica Biblioteca y Sociedad (17\%) con objeto de acercar la biblioteca a la sociedad, garantizando a los usuarios y al conjunto de los ciudadanos el acceso a la información, al conocimiento científico y a la cultura. Esta labor social queda patente en los proyectos de cooperación bibliotecaria en países subdesarrollados y en la mejora de los servicios e infraestructuras para 
las personas de avanzada edad así como para los discapacitados físicos. Seguidamente, se puede citar con un $14 \%$ la línea estratégica Organización y Calidad, que ha sido seleccionada en cuatro ocasiones. Algunos de estos proyectos, como la actualización del plan de mercadotecnia de la biblioteca o el acto de bienvenida para los alumnos de nuevo ingreso en la facultad, inciden en el fortalecimiento de las políticas o sistemas internos de calidad de las unidades de información y documentación.

Por último, queda patente en los resultados la presencia de las líneas estratégicas Financiación e Infraestructuras y Colecciones. En el primer caso $(10 \%)$ los proyectos están relacionados con la gestión de los recursos económicos y físicos (vinculado a los proyectos de teletrabajo o la creación de un repositorio informacional), mientras que la segunda línea estratégica únicamente se ha seleccionado en la formulación del proyecto de donación de libros (3\%).

\section{Los stakeholders o grupos de interés de los proyectos}

En términos generales, los resultados obtenidos presentan seis categorías de stakeholders (Gráfico 2), entre los que destaca la propia Universidad como grueso del conjunto con una representación que supone un $70 \%$, aproximadamente.

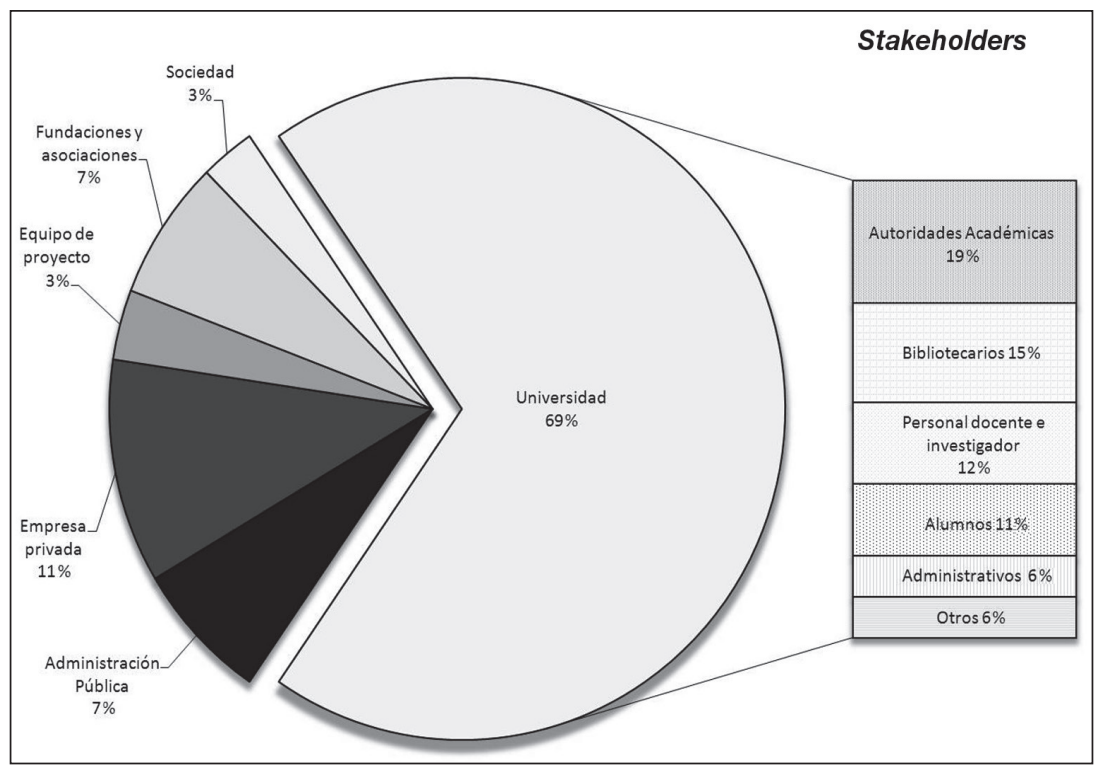

Gráfico 2. Identificación de los stakeholders en los proyectos propuestos por los alumnos. 
Dada la magnitud de este tipo de stakeholder se ha considerado conveniente identificar los agentes que forman el conjunto universitario así como el grado de participación de estos actores. Como se puede observar en la parte derecha del gráfico, los substakeholders que constituyen el colectivo "Universidad" son fundamentalmente autoridades académicas, bibliotecarios, personal docente e investigador y el alumnado. La empresa privada se sitúa en segunda posición (11\%), frente a la administración y al conjunto "Fundaciones y asociaciones" que ocupan el tercer puesto, alcanzando ambos un $7 \%$. En algunos casos han sido identificados como stakeholders implicados en la financiación y en el apoyo de los proyectos. También es importante señalar la repercusión de los proyectos bibliotecarios en el conjunto de la sociedad y la implicación de equipo de trabajo (3\%) al ser éstos los beneficiarios directos de su implementación y los responsables de su gestión, respectivamente.

\section{La viabilidad de los proyectos en términos de tiempo y costes}

Las estimaciones de tiempo para la gestión de los proyectos propuestos en CoP-Innova se han calculado tanto en días como en horas, adoptando como criterio general una duración de 20 días laborables al mes y jornadas de 8 horas. Como marco global de la duración de los proyectos ha de tenerse en cuenta que en los requerimientos temporales fijados en el marco de la CoP se estableció que los proyectos debían proponerse para el alcance temporal de un plan operativo, es decir, para el alcance temporal máximo de un ejercicio contable anual.

Teniendo en cuenta dicha referencia, se ha considerado necesario el establecimiento de 3 niveles o grados de duración según un determinado rango de días: duración baja (propuestas de gestión inferiores a 100 días), duración media (proyectos con una temporalidad comprendida entre 100 y 200 días) y duración alta (proyectos estimados en más de 200 días). Los resultados indican que la mayoría de los proyectos tienen una duración media, concretamente 7 formulaciones de planes; la duración baja está representada por 6 propuestas y sólo 4 proyectos son de duración alta.

Los costes son otro factor clave e imprescindible en la gestión de cualquier proyecto, pues no considerarlos implicaría una falta de responsabilidad e incluso la inviabilidad del proyecto iniciado. Para evitar esta situación y controlar las estimaciones presupuestarias de los planes de proyecto se han establecido 3 niveles o tipos de coste: coste bajo (cantidad inferior a $15000 €$ ), coste medio (entre $15000 €$ y $30000 €$ ) y coste alto (estimación superior a $30000 €$ ), representados porcentualmente en el Gráfico 3. Como 
se evidencia, el $47 \%$ de los proyectos presentan un coste elevado para ser implementados, posiblemente por la necesaria adquisición de equipamiento informático, característica común de algunos proyectos agrupados bajo este tipo de coste (es el caso del teletrabajo o la creación de repositorios informacionales). Otra particularidad a señalar es el elevado número de proyectos que presentan un coste bajo, posiblemente por la actual situación de crisis económica, motivo por el que sorprende el reducido número de propuestas estimadas entre $15000 €$ y $30000 €$ (coste medio).

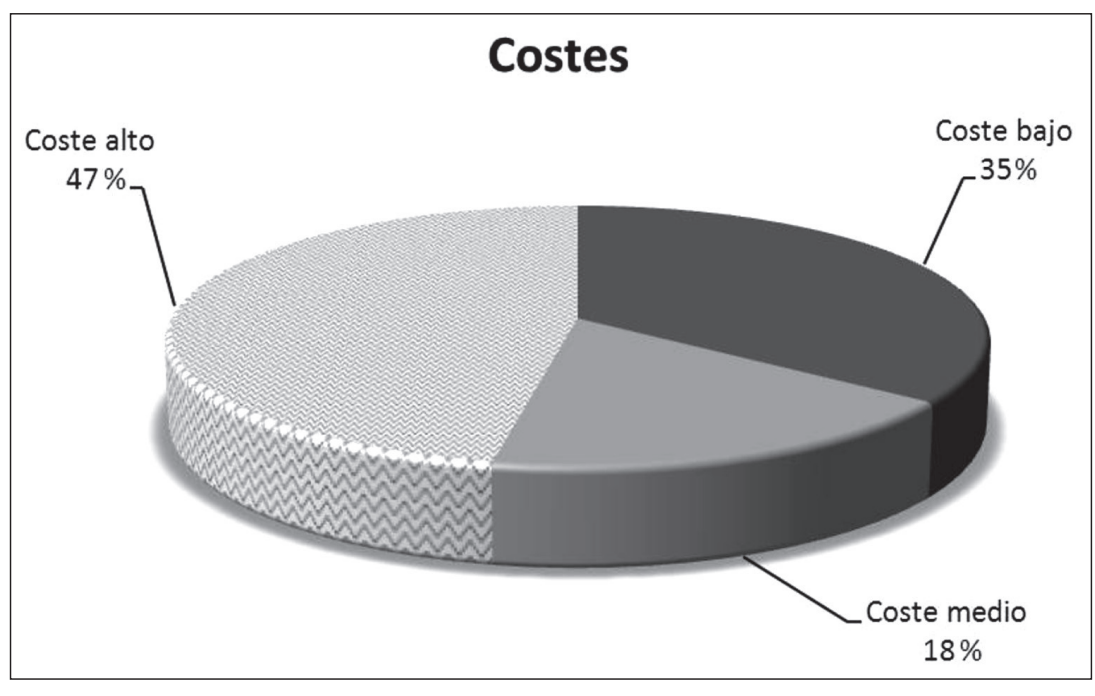

Gráfico 3. Identificación de los costes (análisis en conjunto).

Acorde con estas evidencias, la CoP ha establecido una relación entre las estimaciones temporales y las presupuestarias en la fase de evaluación y discusión de las propuestas finales de proyectos con vistas a su implementación real, de tal manera que la viabilidad de un proyecto se pueda basar en una correspondencia adecuada entre tiempo y coste, siendo conscientes de la repercusión que tiene la crisis económica en la asignación presupuestaria de la unidad de información y documentación, concedida por parte de la organización matriz. Por este motivo, los proyectos con un alto coste suelen tener a priori un alto grado de inviabilidad debido a la dificultad para asumir la inversión. Además, tal y como se ha señalado, la duración de los proyectos no puede ser superior a un ejercicio contable, de tal manera que se pueda determinar el grado de cumplimiento de la planificación operativa anual.

En consecuencia, los proyectos más adecuados presentan un perfil de coste bajo o medio (máximo 30 000€) y una duración baja o media (hasta 200 
días, pues una estimación por curso académico implica una duración de 9 meses con 20 días laborables al mes, lo que supone un total de 180 días).

De acuerdo con esta premisa, los proyectos con mayor grado de viabilidad son los que se muestran a continuación.

Tabla 1. Relación de proyectos viables según la relación coste-duración.

\begin{tabular}{|c|c|c|c|c|c|}
\hline \multirow{2}{*}{$\begin{array}{c}\text { ID } \\
\text { Grupo }\end{array}$} & \multirow{2}{*}{ Título abreviado } & \multicolumn{3}{|c|}{ Estimaciones } \\
\cline { 3 - 6 } & & \multicolumn{2}{|c|}{ Coste } & \multicolumn{2}{c|}{ Duración } \\
\hline 002 & Evaluación de puestos de trabajo & Bajo & $\boldsymbol{U}$ & Media & $\boldsymbol{\partial}$ \\
\hline 004 & Minusvalía auditiva & Bajo & $\boldsymbol{U}$ & Media & $\boldsymbol{\vartheta}$ \\
\hline 008 & Netvibes & Medio & $\boldsymbol{\vartheta}$ & Media & $\boldsymbol{\vartheta}$ \\
\hline 010 & Rotación de puestos de trabajo & Bajo & $\boldsymbol{U}$ & Baja & $\boldsymbol{U}$ \\
\hline 014 & Teletrabajo & Bajo & $\boldsymbol{U}$ & Baja & $\boldsymbol{U}$ \\
\hline 015 & Marketing & Medio & $\boldsymbol{\vartheta}$ & Media & $\boldsymbol{\vartheta}$ \\
\hline 017 & Relaciones Públicas & Bajo & $\boldsymbol{U}$ & Baja & $\boldsymbol{U}$ \\
\hline
\end{tabular}

\section{CONCLUSIONES Y PROYECCIÓN DE LA EXPERIENCIA}

Se ha constituido una comunidad de práctica basada en el desarrollo de ideas innovadoras para la planificación y gestión de proyectos como apoyo a la mejora de la calidad de las bibliotecas universitarias: la Biblioteca de Ciencias Económicas y Empresariales de la Universidad Complutense de Madrid, elegida como escenario piloto por ser Biblioteca Coordinadora del Campus de Somosaguas de la Universidad Complutense

Desde una perspectiva global, CoP-Innova ha estado integrada por grupos de personas que han compartido necesidades y experiencias con un mismo interés, unos formándose y entrenándose (alumnos del Máster Universitario en Gestión de la Documentación, Bibliotecas y Archivos de la Facultad de Ciencias de la Documentación) y otros evaluando sus propuestas y aportando directrices basadas en el método y la experiencia profesional (profesores de la Facultad de Ciencias de la Documentación y profesionales del ámbito de la consultoría en Información y Documentación y de la biblioteca piloto de la Facultad de Ciencias Económicas y Empresariales).

De esta experiencia se pueden extraer varias claves: es una dinámica que facilita la motivación y la participación; disponer de un escenario piloto permite la aplicación de las bases teóricas a un contexto real y, por tanto, una mejor asimilación y comprensión de los contenidos impartidos; la formulación de iniciativas en forma de proyectos genera un clima de emprendedores muy cercano a las demandas de la sociedad actual y, por último, los profesionales 
pueden reutilizar las propuestas con ánimo de mejorar sus centros y con el conocimiento previo del estudio de su viabilidad.

CoP-Innova ha supuesto una experiencia pionera en España en formación e investigación acerca de planificación y gestión de proyectos en bibliotecas universitarias. Se ha generado un directorio de proyectos innovadores para el contexto de la biblioteca piloto mencionada y se ha desarrollado una experiencia real de participación integradora de actores de los diferentes sectores de la universidad: alumnos, personal bibliotecario, personal docente e investigadores.

Las encuestas de calidad de la asignatura realizadas en la Facultad de Ciencias de la Documentación de la Universidad Complutense han constatado una mejora de la calidad del proceso de aprendizaje de los alumnos en el marco de los objetivos de la asignatura mencionada y sobre la base del principio básico de una comunidad de práctica: todos tienen algo que enseñar y todos tienen algo que aprender.

Se ha realizado una experiencia de inmersión de los alumnos en un escenario real de pilotaje profesional, partiendo del conocimiento de los principios teóricos y metodológicos para la planificación y gestión de proyectos en unidades de información y documentación.

Finalmente, pensamos que sobre la base metodológica presentada y sobre el estudio de caso realizado se pueden derivar nuevas aplicaciones y proyecciones en otros escenarios de innovación y emprendimiento que contemplen propuestas de proyectos:

En tipologías adicionales de unidades de información y documentación, además de las bibliotecas universitarias, con el fin de explorar diferencias y similitudes en el desarrollo y resultados de la experiencia.

En relación con otras materias y/o líneas de investigación en el área de Bibliotecología y Ciencias de la Documentación.

\section{BibLIOgRAFÍA}

Adeyoyin, S. O. \& Akinyosoye, H. I. T. (2004), "Retrospective conversion in a Nigerian research library: Migrating from TINMAN/ TINLIB to CDS/ISIS”, The Electronic Library, 22 (4), 331-334.

Allan, B. (2004), Project management: Tools and techniques for today's ILS professional, London: Facet.

Anderson, D. R. (1998), "Method without madness: Shelf-reading methods and project management", College and Undergraduate Libraries, 5 (1), 1-13.

Anzalone, F. M. (2000), "Project management: A technique for coping with change”, Law Library Journal, 92 (1), 53-70. 
Arquero, R. \& García-Ochoa, M. L. (2005), "Planificación de hemerotecas de prensa”, en La bemeroteca de prensa, Madrid: Trea, 31-45.

Atkinson, R. (1999), "Project management: Cost, time and quality, two best guesses and a phenomenon, its time to accept other success criteria”, International Journal of Project Management, 17 (6), 337-342.

Barwick, J. (2007), "Building an institutional repository at Loughborough University: Some experiences”, Program: Electronic Library and Information Systems, 41 (2), 113-123.

Betty, P. (2008), "Creation, management, and assessment of library screencasts: The Regis Libraries Animated Tutorials Project", Journal of Library Administration, 48 (3-4), 295-315.

Biblioteca de la Universidad Complutense de Madrid (2010), Plan Estratégico 2010-2013. Disponible en: http://www.ucm.es/BUCM/ intranet/doc14417.pdf (Fecha de consulta: 13 de julio de 2012).

_ (2013), Nuestras bibliotecas. Disponible en: http://www.ucm.es/ BUCM/biblioteca/5762.php (Fecha de consulta: 5 de mayo de 2013).

Black, K. (1996), Project management for library and information service professionals, London: Aslib.

Brown, J. \& Duguid, P. (1991), "Organizational learning and communities of practice: toward a unified view of working, learning, and innovation”, Organizational Science, 2 (1), 40-57.

Bryson, J. (1997), Managing information services: An integrated approach, Aldershot: Gower.

- (1999), Effective library and information centre management, 2a ed., Aldershot, England: Gower.

Bueno Campos, E. (1996), Dirección estratégica de la empresa: metodología, técnica y casos, Madrid: Pirámide.

Burich, N. J.; Casey, A. M.; Devlin, F. A. \& Ivanitskaya, L. (2006), "Project management and institutional collaboration in libraries", Technical Services Quarterly, 24 (1), 17-36. DOI:10.1300/J124v24n 01_02

Carpenter, J. (2008), Library project funding: A guide to planning and writing proposals, Oxford: Chandos.

- (2011), Project management in libraries, archives and museums: Working with government and other external partners, Oxford: Chandos.

Chellapandi, S.; Han, C. W. \& Boon, T. C. (2010), “The National Library of Singapore experience: Harnessing technology to deliver content and broaden access", en Interlending E Document Supply, 38 (1), 40-48.

Clements, C. (2009), "Implementing instant messaging in four university libraries”, Library Hi Tech, 27 (3), 393-402.

Darko-Ampem, K. (2006), "Retrospective conversion of serials and card catalogue records: A case study of project management in academic libraries", Library Management, 27 (3), 121-134. 
DeRidder, J. L. (2007), "Choosing software for a digital library", $L i$ brary Hi Tech News, 24 (9/10), 19-21.

Duncan, V. \& Gerrard, A. (2011) "All together now!: Integrating virtual reference in the academic library", Reference \& User Services Quarterly, 50 (3), 280-292.

Drucker, P. (2002), La Gerencia: tareas, responsabilidades y prácticas, Buenos Aires: El Ateneo.

European Ministers of Education (1999), The european higher education area. Joint declaration of the European Ministers of Education convened in Bologna, 19 June 1999. Disponible en: http://www.ugent. be/nl/univgent/reglementen/internationaal/bologna.pdf (Fecha de consulta: 16 de julio de 2012).

Evans, E. R. (2011), An investigation into the extent and application of bilingual signage in New Zealand public libraries (Unpublished School of Information Management, Victoria University of Wellington, Wellington, New Zealand).

Evans, G. E. \& Layzell Ward, P. (2003), Beyond the basic: The management guide to library and information professionals, New York: Neal-Schuman.

Evans, M. F. \& Thomas, S. (2007), "Implementation of an integrated information management system at the National Library of Wales: A case study", Program: Electronic Library and Information Systems, 41 (4), 325-337. DOI:10.1108/00330330710831558

Fagan, J. C. (2009), Web project management for academic libraries, Oxford: Chandos.

Fenton, C. (2007), "Finding the way: Improving access to the collections of the Royal Scottish Geographical Society", Program: Electronic Library and Information Systems, 41 (4), 353-364.

Gongla, P. \& Rizzuto, C. (2001), "Evolving communities of practice: IBM Global Services experience", IBM Systems Journal, 40 (4), 842-862.

Greene, J. (2010), "Project management and institutional repositories: A case study at University College Dublin Library", New Review of Academic Librarianship, 16, 98-115.

Handley, K.; Sturdy, A.; Fincham, R. \& Clark, T. (2006), "Withing and beyond communities of practice: making sense of learning through participation, identity and practice", Journal of Management Studies, 43 (3), 641-53.

Hansen, M. T. \& Birkinshaw, J. (2007), "La cadena de valor en la innovación”, Harvard Business Review, 85 (6), 100-110.

Hayman, L. M. (1997), "Database design for preservation project management: The California Newspaper Project", Library Resources and Technical Services, 41 (3), 236-253.

Henshaw, C. (2011), "The Wellcome Digital Library: Building a sustainable infrastructure", Library Hi Tech News, 28 (1), 18-21. 
Holley, R. (2004), "Developing a digitisation framework for your organisation”, Electronic Library, 22 (6), 518-522.

Holly, M. L. (2004), "Learning in Community: Small Group Leadership for Educational Change", Educar, 34, 113-130.

Hull, R. W. \& Dreher, S. L. (2001), “ 'Into the middle of the thing' (with apologies to Horace): Developing a system to manage a grant-funded digital collection project", Collection Management, 26 (3), 29-38.

Koontz, C. M.; Jue, D. K.; McClure, C. R. \& Bertot, J. C. (2004), “The Public Library Geographic Database: What can it do for your library?", Public Libraries, 43 (2), 113-118.

Koontz, H. \& O’Donnell, K. (1972), Principles of Management, New York: McGraw-Hill.

_ \& Weihrich, H. (1994), Administración: Una perspectiva global, México: McGraw-Hill.

Lesser, E. \& Everest, K. (2001), "Using communities of practice to manage intellectual capital”, Ivey Business Journal, March-April, 37-41.

— \& Starck, J. (2001), "Communities of practice and organizational performance”, IBM Systems Journal, 40 (4), 831-841.

Levi, P. (2010), "Digitising the past: The beginning of a new future at the Royal Tropical Institute of the Netherlands", Program: Electronic Library and Information Systems, 44 (1), 39-47.

Lewis, A. C. (1995), "The use of PRINCE project management methodology in choosing a new library system at the University of Wales Bangor", Program: Electronic Library and Information Systems, 29 (3), 231-240.

Londhe, N. L.; Desale, S. K. \& Patil, S. K. (2011), "Development of a digital library of manuscripts: A case study at the University of Pune, India", Program: Electronic Library and Information Systems, 45 (2), 135-148.

Lynch, B. P. (1985), Management strategies for libraries: A basic reader, New York: Neal-Schuman.

MacLachlan, L. (1996), Making project management work for you, England: Library Association.

Martey, A. K. (2002), "Management issues in library networking: Focus on a pilot library networking project in Ghana", Library Management, 23 (4/5), 239-252.

McClure, C. R. (1982), Strategies for library administration: Concepts and approaches, Littleton, Colorado: Libraries Unlimited.

Medawar, K. (2007), "The restructuring of the portal at the Institut Pasteur Library: A case study", en Program: Electronic Library and Information Systems, 41 (1), 20-34. DOI:10.1108/00330330710724863

Middleton, M. R. (1999), Library digitisation project management, 20th IATUL (International Association of Technological University Libraries) Conference, May, 1999, Chania, Greece, 1-7.

Moore, K. (1998), "Project management: Can libraries benefit?”, Bibliotheca Medica Canadiana, 20 (2), 72-73. 
Müller, H. \& Maurer, H. (2011), "How to carry over historic books into social networks", Proceedings of the 4th ACM Workshop on Online Books, Complementary Social Media and Crowdsourcing, New York: ACM, 25-34. DOI: 10.1145/2064058.2064065

Murphy, E. (2008), "Delivering core text material electronically: A project management perspective”, en SCONUL Focus, (43), 29-32.

Otunla, A. O. \& Akanmu-Adeyemo, E. A. (2010), "Library automation in Nigeria: The Bowen University experience", African Journal of Library, Archives and Information Science, 20 (2), 93-102.

Pacios, A. R. (1997), "La administración de las unidades de información: una aproximación a su concepto y evolución”, en Documentación de las Ciencias de la Información, 20,225-248.

Palincsar, A. \& Herrenkohl, L. (2002), "Designing collaborative learning contexts", Theory into Practice, 41 (1), 26-32.

Pan, S. \& Leidner, D. (2003), "Bridging communities of practice with information technology in pursuit of global knowledge sharing", Journal of Strategic Information System, 12 (1), 71-88.

Piorun, M. \& Palmer, L. A. (2008), "Digitizing dissertations for an institutional repository: A process and cost analysis", Journal of the Medical Library Association, 96 (3), 223-229.

Prytherch, R. (1994), Information management and library science: A guide to the literature, Aldershot, England: Gower.

Ramos, L. F. (1995), Dirección, administración y marketing de empresas e instituciones documentales, Madrid: Síntesis.

— (2003), Introducción a la administración de información, Madrid: Síntesis.

Roberts, J. (2006), "Limits to communities of practice", Journal of Management Studies, 43 (3), 623-639.

Rosacker, K. M. (2010), "Information technology project management within public sector organization", Journal of Enterprise Information Management, 23 (5), 587-594.

Roselló, D. (2006), "La aventura de leer: El fomento de la biblioteca pública como espacio para la lectura”, en Lluís Bonet, Xavier Castañer y Josep Font Sentias (coords.), Gestión de proyectos culturales: Análisis de casos, Barcelona: Ariel, pp. 99-123.

Saikia, R. R. \& Kalita, B. (2011), "Prospects of digitizing manuscript collections in KKH library: a model", 8th International CALIBER, Goa: Goa University, March 2-4,312-319.

Sakaguchi, E.; Shimada, K. \& Wasserstrom, A. (2010), "A cross-Pacific partnership: The University of Maryland Libraries and the National Diet Library of Japan jointly reformat children's books", OCLC Systems E Services, 26 (1), 18-28.

Sharpe, P. A. \& Vacek, R. E. (2010), "Intranet 2.0 from a project management perspective", Journal of Web Librarianship, 4 (2-3), 239-249.

Stephenson, W. R. (1991), "Seminar on project management for libraries/documentalists", Guyana Library Association Bulletin, 20 (2), 46-52. 
Stueart, R. D. \& Moran, B. B. (2002), Library and information center management, Englewood: Libraries Unlimited.

Train, B. \& Elkin, J. (2001), "Effecting change: Reader development projects in public libraries", Library Management, 22 (8/9), 395-403.

Universidad Complutense de Madrid (2002), Universidad Complutense de Madrid: de la Edad Media al III Milenio, Madrid: Editorial Complutense.

— (2011), Convocatoria de proyectos de innovación y mejora de la calidad docente. Disponible en: http://www.ucm.es/: http://www. ucm.es/cont/descargas/documento37379.pdf (Fecha de consulta: 9 de julio de 2012).

_ (2013), Universidad Complutense de Madrid: web oficial. Disponible en: http://www.ucm.es/?a=directorio/dap\&d=0024286 (Fecha de consulta: 21 de mayo de 2013).

Vásquez, S. (2011), “Comunidades de práctica”, Educar, 47 (1), 51-68.

Wagner, P. (2006), "The three skills you need to have for successful project management”, Information Outlook, 10 (8), 24-26.

Webb, Sylvia (2001), "Project management in library and information services”, Information Management Report, 16-19.

Wenger, E. (1998), Communities of practices: learning, meaning, and identity, Cambridge: Cambridge University Press.

Wenger, E. \& Lave, J. (1991), Situated learning: legitimate peripheral participation, Cambridge: Cambridge University Press.

Winston, M. D. \& Hoffman, T. (2005), "Project management in libraries”, Journal of Library Administration, 42 (1), 51-61. 
Apéndice

Directorio de proyectos propuestos

Se presenta en este apéndice la síntesis del directorio de los 17 proyectos resultantes de la experiencia desarrollada en CoP-Innova, que responde a la siguiente estructura-tipo: identificador de proyecto/grupo, título del proyecto propuesto y línea estratégica del Plan Estratégico 2010-2013 de la Biblioteca de la Universidad Complutense en la que se engloba.

\begin{tabular}{|c|c|c|}
\hline ID & Título & Línea estratégica \\
\hline 001 & $\begin{array}{l}\text { Teletrabajo de la Biblioteca de Económicas de la Universidad } \\
\text { Complutense de Madrid }\end{array}$ & 1. Personas \\
\hline 002 & $\begin{array}{l}\text { Evaluación de puestos de trabajo de la Biblioteca de Económicas } \\
\text { y Empresariales }\end{array}$ & 1. Personas \\
\hline 003 & Biblioteca y Sociedad & 5. Biblioteca y Sociedad \\
\hline 004 & $\begin{array}{l}\text { Accesibilidad de usuarios con minusvalía auditiva a la Biblioteca } \\
\text { de la Universidad Complutense de Madrid }\end{array}$ & $\begin{array}{l}\text { 1. Personas } \\
\text { 3. Financiación e Infr. } \\
\text { 4. Servicios } \\
\text { 5. Biblioteca y Sociedad }\end{array}$ \\
\hline 005 & Promoción y difusión de los servicios bibliotecarios & 2. Organización y calidad \\
\hline 006 & $\begin{array}{l}\text { Estudio de las necesidades informativas de los usuarios extran- } \\
\text { jeros en la biblioteca de la Facultad de Ciencias Económicas y } \\
\text { Empresariales de la Universidad Complutense de Madrid }\end{array}$ & $\begin{array}{l}\text { 1. Personas } \\
\text { 4. Servicios }\end{array}$ \\
\hline 007 & Repositorio informacional & $\begin{array}{l}\text { 2. Organización y calidad } \\
\text { 3. Financiación e Infr. } \\
\text { 4. Servicios } \\
\text { 5. Biblioteca y Sociedad }\end{array}$ \\
\hline 008 & Propuesta de implantación de la herramienta Netvibes & 4. Servicios \\
\hline 009 & $\begin{array}{l}\text { Programa formativo de bienvenida a la BUC para alumnos de } \\
\text { nuevo ingreso }\end{array}$ & $\begin{array}{l}\text { 1. Personas } \\
\text { 2. Organización y calidad } \\
\text { 4. Servicios }\end{array}$ \\
\hline 010 & $\begin{array}{l}\text { Proyecto de rotación en puestos de trabajo de la Biblioteca de } \\
\text { Ciencias Económicas y Empresariales de la UCM para empleados } \\
\text { de reciente incorporación }\end{array}$ & 1. Personas \\
\hline 011 & $\begin{array}{l}\text { Proyecto bibliotecario en países subdesarrollados y } / 0 \text { en vías de } \\
\text { desarrollo }\end{array}$ & 5. Biblioteca y Sociedad \\
\hline 012 & $\begin{array}{l}\text { Recursos de información, metodología y redes sociales aplica- } \\
\text { dos a las Ciencias Económicas y Empresariales en el EEES }\end{array}$ & 4. Servicios \\
\hline 013 & $\begin{array}{l}\text { "Dona cultura a tu Universidad". Campaña de donación de libros } \\
\text { a la Biblioteca de la Facultad de CC. Económicas y Empresariales } \\
\text { de la UCM }\end{array}$ & $\begin{array}{l}\text { 4. Servicios } \\
\text { 6. Colecciones }\end{array}$ \\
\hline 014 & El bibliotecario en casa & $\begin{array}{l}\text { 1. Personas } \\
\text { 3. Financiación e Infr. }\end{array}$ \\
\hline 015 & Renovación del plan de Marketing & 2. Organización y calidad \\
\hline
\end{tabular}




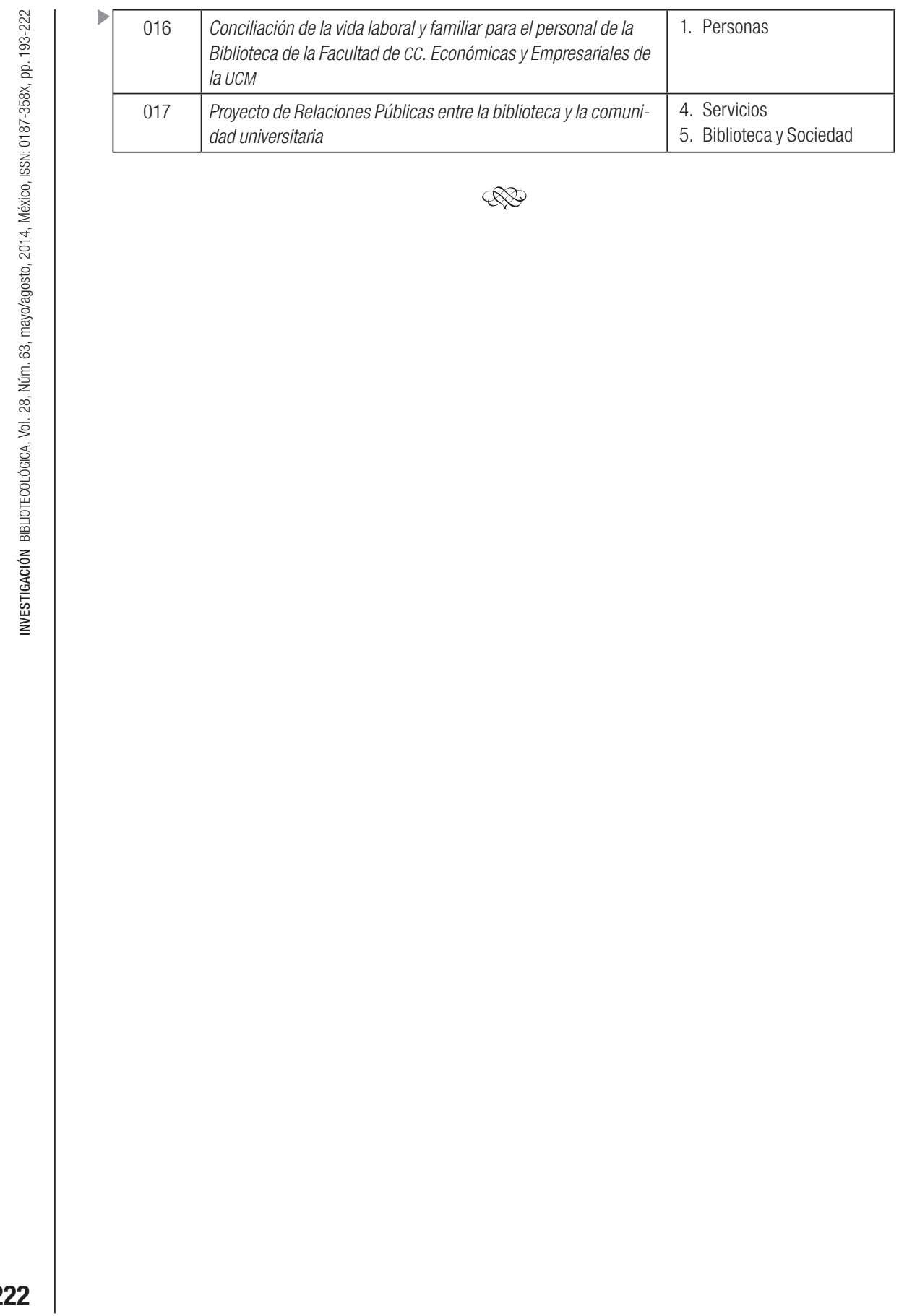

